INSTITUT NATIONAL DE RECHERCHE EN INFORMATIQUE ET EN AUTOMATIQUE

\title{
TCP Throughput Analysis under Transmission Error and Congestion Losses
}

François Baccelli — Ki Baek Kim

$\mathbf{N}^{\circ} 4953$

Octobre 2003

THÈME 1 



\title{
RIN RIA
}

\section{TCP Throughput Analysis under Transmission Error and Congestion Losses}

\author{
François Baccelli*, Ki Baek Kim ${ }^{\dagger}$ \\ Thème 1 - Réseaux et systèmes \\ Projet TREC \\ Rapport de recherche $n^{\circ} 4953$ - Octobre 2003 - 27 pages
}

\begin{abstract}
This paper analyzes the performance of a large population of long lived TCP flows experiencing random packet losses due to both random transmission errors and congestion created by the sharing of a common tail drop bottleneck router. We propose a natural and simple model for the joint throughput evolution of the set of TCP sessions under such a mix of losses. For the case of Poisson transmission errors, we show that the asymptotic model where the population tends to infinity leads to a well defined and tractable dynamical system. In particular, we get the mean value of the throughput of each session as a function of the transmission error rate and the synchronization rate in the bottleneck router. The large population asymptotic model has two interesting and nonintuitive properties:
\end{abstract}

1. there exists a positive threshold (given in closed form) on the transmission error rate above which there are no congestion losses at all in steady state;

2. below this threshold, the mean throughput of each flow is an increasing function of the transmission error rate, so that the maximum mean value is in fact achieved when the transmission error rate is equal to this threshold.

The finite population model and models based on other classes of point processes are also studied. In particular, a sufficient condition is obtained for the existence of congestion times in the case of arbitrary transmission error point processes.

Key-words: TCP, congestion control, flow control, additive increase-multiplicative decrease algorithm, IP traffic, synchronization, throughput, bit error, packet error, transmission error.

\footnotetext{
* INRIA-ENS, 45 rue d'Ulm 75005, Paris, France, francois .baccelli@ens.fr

${ }^{\dagger}$ INRIA-ENS, 45 rue d'Ulm 75005, Paris, France, kkb@di.ens. fr
} 


\section{Analyse du débit de TCP avec des pertes de deux types: pertes dues à des erreurs de transmission et pertes dues à la congestion}

Résumé : Nous étudions dans cet article les performances d'une population formée d'un grand nombre de flots TCP subissant des pertes de paquets aléatoires et de deux types: des pertes dues à des erreurs de transmission et des pertes dues à la congestion résultant du partage d'un routeur commun. Nous proposons un modèle simple et assez naturel pour l'évolution jointe des débits de ces flots TCP sous de telles conditions. Dans le cas où les erreurs de transmission suivent des lois de Poisson, nous montrons que le modèle asymptotique obtenu en faisant tendre la taille de la population vers l'infini conduit à un système dynamique bien défini et analysable. Nous donnons en particulier une forme explicite pour le débit stationnaire moyen de chaque flot en fonction du taux des erreurs de transmission et du taux de synchronisation du routeur partagé. Ce modèle asymptotique possède deux propriétés intéressantes et non-intuitives:

1. Il existe un seuil positif (donné explicitement) sur le taux des erreurs de transmission au dessus duquel il n'y a pas de pertes de congestion en régime stationnaire;

2. En dessous de ce seuil, le débit moyen de chaque flot est une fonction croissante du taux des erreurs de transmission, si bien que le débit optimal est obtenu lorsque le taux des erreurs de transmission est égal au seuil.

Le modèle avec population finie et ceux fondés sur d'autres classes de processus ponctuels sont aussi étudiés. En particulier, nous donnons une condition suffisante d'existence de pertes par congestion valable pour tout modèle, indépendamment de la taille de la population et du type des processus ponctuels des pertes par erreur de transmission.

Mots-clés : TCP, contrôle de congestion, contrôle de flux, algorithme de croissance additive et décroissance multiplicative, trafic IP, synchronisation, débit, erreur par bit, erreur par paquet, erreur de transmission. 


\section{Introduction}

Understanding the behavior of TCP in the presence of random transmission errors has become important with the current increase of the proportion of wireless and of DSL access links in the Internet, where bit/packet error rates are essential features.

The present paper studies the interaction of TCP flows experiencing packet losses due to random transmission errors in addition to losses created by congestion, under the assumption that the flows share a common tail drop bottleneck router/link.

Previous studies on TCP over hybrid wired/wireless links have primarily focused on improving the performance by hiding or reducing packet losses due to random transmission errors [1], [2]. Among the main ideas along these lines, we would quote

- the addition of mechanisms that allow TCP to identify and to ignore packet losses due to random transmission errors [3], [4], [5];

- the reduction of such packet losses obtained either by FEC (Forward Error Correction), or by breaking the end-to-end connection into two parts (one from the mobile to the base station and one from the base station to the destination) [6], [7].

For all situations where TCP has to cope with some bit/packet error rate, no formula seems to be available for characterizing the interplay between these transmission error losses and losses due to congestion. Our aim in the present paper is to analyze this interplay and to determine the throughput obtained by each flow from the knowledge of the RTT, the capacity of the shared outer/link and random loss rate.

This work builds upon the AIMD (Additive Increase, Multiplicative Decrease) model introduced in $[8,9]$ for describing the interaction of TCP flows over wired links $([8,9])$. The main new feature of the present paper is the addition of random transmission errors. This new model, which will be referred to as the transmission error-AIMD model, is introduced in $\S 2$.

In $\S 3$, we analyze the dynamics of this model under the assumption of a large population of long lived TCP flows experiencing Poisson transmission errors. This large population asymptotic model is then used in $\S 4$ in order to derive the mean value of the throughput of each session as a function of the transmission error rate and the synchronization rate in the bottleneck router. We also show that the obtained throughput formula is actually a refinement to the classical square root formula for TCP throughput [10], [11].

In $\S 5$, the finite population model and models based on other classes of point processes are also studied. A sufficient condition is obtained for the existence of congestion times in the case of arbitrary transmission error point processes.

Finally, in $\S 6$, we illustrate the two main conclusions of this paper for the large population asymptotic model in the case of Poisson transmission errors, and show that these two conclusions hold for finite population models and other classes of point processes. 


\section{The Transmission Error-AIMD Model}

In this section, we propose a set of fluid evolution equations allowing one to represent the key features of the AIMD mechanism for $N$ homogeneous TCP sessions sharing one tail drop bottleneck router in the presence of random packet losses due to transmission errors. We will first consider the homogeneous case, where all sessions have the same RTT. We will also give the equations for the heterogeneous case (different RTT's or synchronization rates). These equations will not be studied in the present paper.

\subsection{Notation}

By definition, the $n$-th congestion time is the $n$-th epoch at which a loss or several simultaneous losses occur due to congestion on this shared router. We will use the following notation:

- $N$ is the number of TCP sessions, which we assume to be constant with time;

- $C=c N$ is the capacity of the bottleneck router;

- $T_{n}^{N}$ is $n$-th congestion time, namely the $n$-th epoch at which there is one of more losses due to congestion on the router. This quantity is not known in advance and will have to be determined from the analysis; it may even be the case that there are less than $n$ congestion epochs (in which case we take $T_{n}^{N}=\infty$ by convention);

- $\tau_{n+1}^{N}=T_{n+1}^{N}-T_{n}^{N}$ whenever $T_{n}^{N}$ is finite. By convention, we take $\tau_{n+1}^{N}=\infty$ whenever $T_{n}^{N}$ is infinite;

- $X_{n}^{i, N}$ is the throughput of session $i$ just after the $n$-th congestion time;

- $W_{n}^{i, N}$ is the window size of session $i$ just after the $n$-th congestion time;

- $X_{n}^{i, N}(t)$ is the throughput of session $i$ at time $T_{n}^{N}+t$, for $t \in\left[0, \tau_{n+1}^{N}\right]$ (all jump functions will be assumed right continuous; we have in particular $X_{n}^{i, N}(0)=X_{n}^{i, N}$ );

- $L_{n}^{i, N}(t)$ is the total throughput drop of session $i$ from $T_{n}^{N}$ to $T_{n}^{N}+t$ due to the packet transmission losses, with $t \in\left[0, \tau_{n+1}^{N}\right]$;

- $R^{(i)}$ is the mean RTT of session $i$ (in the homogeneous situation, $R^{(i)}=R$ );

- $\alpha^{(i)}$ is the linear growth rate of the window size of session $i$ with time; it makes sense to take $\alpha^{(i)}=1 / R^{(i)}$;

- $\gamma_{n}^{(i)}$ is a $\left\{\frac{1}{2}, 1\right\}$-valued random variable with value $\frac{1}{2}$ if session $i$ experiences a loss at the $n$-th congestion time, 1 otherwise;

- $\delta_{n}^{(i)}(t)$ is a non-negative integer valued random variable with value $l$ if session $i$ experiences $l$ packet transmission losses from $T_{n}^{N}$ to $T_{n}^{N}+t, t \geq 0$. 
We will also use the following aggregated quantities:

$$
S_{n}^{N}(t)=\sum_{i=1}^{N} X_{n}^{i, N}(t), \quad M_{n}^{N}(t)=\sum_{i=1}^{N} L_{n}^{i, N}(t)
$$

\subsection{Dynamics}

We are now in a position to give the equations of the dynamics of our model. Assuming that $T_{n}^{N}$ is finite, the throughput of session $i$ evolves according to the following law:

$$
X_{n}^{i, N}(t)=X_{n}^{i, N}+\frac{\alpha^{(i)}}{R^{(i)}} t-L_{n}^{i, N}(t),
$$

for $t \in\left[0, \tau_{n+1}^{N}\right)$. The rationale for this equation is as follows:

- one assumes that TCP Reno is used and that all sources are in the congestion avoidance phase, so that the window has a linear increase of $\alpha^{(i)}=1 / R^{(i)}$;

- one assumes that the throughput and the window size are linked by a Little like law: $W_{n}^{i, N}=$ $X_{n}^{i, N} R^{(i)}$ (which is a simplifying assumption since this way of linking throughput to window only applies to stationary means whereas we use it for linking instantaneous values here).

Then the linear increase of the window leads to a linear increase of the throughput too, with slope $\frac{\alpha^{(i)}}{R^{(i)}}$.

We now define $\tau_{n+1}^{N}$ as

$$
\inf \left\{t>0 \text { s.t. } \sum_{i=1}^{N}\left(X_{n}^{i, N}-L_{n}^{i, N}(t)\right)+\sum_{i=1}^{N} \frac{\alpha^{(i)}}{R^{(i)}} t=C\right\}
$$

with $\tau_{n+1}^{N}=\infty$ if the last set is empty. This defines $T_{n+1}^{N}=T_{n}^{N}+\tau_{n+1}^{N}$. The rationale for this definition stems from the assumption that the buffer capacity of the router is 0 or negligible (see the paper [8] for a simple way to relax this assumption), so that the next congestion takes place at the next time when the sum of all throughputs reach again the capacity $C$ of the router.

There are now two cases: either $\tau_{n+1}^{N}=\infty$ and we have actually constructed the whole dynamics; or $\tau_{n+1}^{N}<\infty$ in which case one defines

$$
X_{n+1}^{i, N}=\gamma_{n+1}^{(i)}\left(X_{n}^{i, N}+\frac{\alpha^{(i)}}{R^{(i)}} \tau_{n+1}^{N}-L_{n}^{i, N}\left(\tau_{n+1}^{N}\right)\right) .
$$

The rationale for this should be clear as by the multiplicative rule of Reno, the throughput of session $i$ should be divided by 2 if this session experiences a loss at $T_{n+1}^{N}$.

Let us now give the value of the $L_{n}^{i, N}(t)$ function. If session $i$ experiences $l$ packet losses, the throughput drop $\left.L_{n}^{i, N}(t)\right|_{l}$ due to transmission losses can be represented as

$$
\left.L_{n}^{i, N}(t)\right|_{l}=X_{n}^{i, N}\left(1-\frac{1}{2^{l}}\right)+\frac{\alpha(i)}{R(i)} \sum_{k=1}^{l+1} \tau_{k}^{i, N}\left(1-\frac{1}{2^{l-k+1}}\right),
$$


where $\tau_{k}^{i, N}, k \leq l$ is the length of the interval from the $k-1$-st transmission loss time to the $k$-th and $\tau_{l+1}^{i, N}$ is the length of the interval from the $l$-th transmission loss time to $t$. The derivation of (5) is given in $\$ 8.1$ in the appendix.

For a validation of this kind of fluid dynamics and its comparison with NS based simulation, the interested reader should consult [8] and [12] for the particular case without transmission errors and $\S 6$ for the case considered here.

\section{Mean Field Asymptotics of the Poisson Transmission Error, Rate Independent Synchronization Case}

The mathematical derivations of this section focus on case with

1. Rate independent synchronization: the random variables $\left\{\gamma_{n}^{(i)}, i=1, \ldots, N\right\}$ are independent of the past throughput processes before time $T_{n}^{N}$ and $\mathbb{P}\left(\gamma_{n}^{(i)}=\frac{1}{2}\right)=p^{(i)}$ for all $n$ (with $p^{(i)}=p$ in the homogeneous case). In the homogeneous case, a simple instance is that where a subset of cardinality $p N$ is selected at random among the $N$ sessions for determining which sessions experience a loss at $T_{n}^{N}$. Other random models are considered in [8]. The parameter $p$ is referred to as the synchronization rate; this parameter describes the proportion of flows that simultaneously experience a loss during a congestion epoch. For queueing theory estimates of this parameter, see [8].

2. Poisson transmission errors: the point processes of packet transmission errors are homogeneous Poisson point processes with intensity $\lambda^{(i)}$ for session $i$ (with $\lambda^{(i)}=\lambda$ in the homogeneous case). Since $T_{n}^{N}$ is a stopping time of this family of Poisson point processes and of the family of random variables $\left\{\gamma_{k}^{(i)}\right\}_{k<n, i=1, \ldots, N}$, then thanks to the Strong Markov property for Poisson point processes [13], whenever $T_{n}^{N}$ is finite, the transmission error point processes after time $T_{n}^{N}$ are independent Poisson point processes, independent of the past throughput processes before time $T_{n}^{N}$. In particular, for all $t$,

- $\delta_{n}^{(i)}(t)$ has a Poisson distribution with parameter $\lambda^{(i)} t$ :

$$
\mathbb{P}\left(\delta_{n}^{(i)}(t)=l\right)=q_{l}^{(i)}=\frac{\left(\lambda^{(i)} t\right)^{l}}{l !} e^{-\lambda^{(i)} t},
$$

with $q_{l}^{(i)}=q_{l}$ in the homogeneous case.

- the random variables $\left\{\delta_{n}^{(i)}(t), i=1, \ldots, N\right\}$ are independent in $i$ and are independent of the past of the throughput processes before time $T_{n}^{N}$.

Note that when $N$ is finite, under the foregoing Poisson assumptions, the random variable $\tau_{n+1}^{N}$ defined in (3) is almost surely (a.s.) finite and even of finite mean. This follows from the fact that for all $a>0$, there is a finite random and integrable stopping time $T$ for which all $N$ Poisson point processes simultaneously have not points at all in the interval $[T, T+a]$. If one takes $a$ large enough for having $\sum_{i=1}^{N} \frac{\alpha^{(i)}}{R^{(i)}} a>C$, then one sees that $\tau_{n+1}^{N} \leq T<\infty$, which concludes the proof. 


\subsection{Homogeneous Case}

From Assumption 1 above and from (4)

$$
\begin{aligned}
\mathbb{E}\left[X_{n+1}^{i, N}\right] & =\mathbb{E}\left[\gamma_{n+1}^{(i)}\right] \mathbb{E}\left[X_{n}^{i, N}-L_{n}^{i, N}\left(\tau_{n+1}^{N}\right)+\frac{\alpha}{R} \tau_{n+1}^{N}\right] \\
& =\left(1-\frac{p}{2}\right) \mathbb{E}\left[X_{n}^{i, N}-L_{n}^{i, N}\left(\tau_{n+1}^{N}\right)+\frac{\alpha}{R} \tau_{n+1}^{N}\right]
\end{aligned}
$$

So

$$
\begin{aligned}
\sum_{i} \mathbb{E}\left[X_{n+1}^{i, N}\right] & =\left(1-\frac{p}{2}\right) \mathbb{E}\left[\sum_{i}\left(X_{n}^{i, N}-L_{n}^{i, N}\left(\tau_{n+1}^{N}\right)+\frac{\alpha}{R} \tau_{n+1}^{N}\right)\right] \\
& =\left(1-\frac{1}{2} p\right) C .
\end{aligned}
$$

Using now the homogeneity assumption, we get that for all $n \geq 1$,

$$
\mathbb{E}\left[X_{n}^{i, N}\right]=\left(1-\frac{p}{2}\right) c .
$$

In order to go further, namely to determine the mean value of the throughput obtained by a flow in continuous time, we now introduce the large population asymptotic model.

\subsection{Large Population Asymptotics}

The next theorem is the main structural result of the paper. It is in the continuation of results used in [14] and [9] and is related to the mean field method of statistical physics (for more on the matter, see the references in [9]).

This theorem is based on conditions using certain subsets $\sigma^{N}$ of $\{1, \ldots, N\}$. The cardinal of set $s$ will be denoted by $|s|$ throughout the paper. We will say that a subset is independent of the throughput process before time $t$ if the elements of the subset are selected according to a random (or deterministic) procedure that is independent of the values of the throughput process before time $t$.

Theorem 1 Assume the initial condition $X_{0}^{i, N}$ is such that

- $\mathbb{E}\left[X_{0}^{i, N}\right]=x_{0}<c$, for all $i$ and

- for all sequences of subsets $\sigma^{N}$ of $\{1, \ldots, N\}$, with a cardinal $\left|\sigma^{N}\right|$ that tends to $\infty$, when $N$ goes to $\infty$, the following almost sure (a.s.) limit holds:

$$
\lim _{N \rightarrow \infty} \frac{1}{\left|\sigma^{N}\right|} \sum_{i \in \sigma^{N}} X_{0}^{i, N}=\lim _{N \rightarrow \infty} \frac{1}{\left|\sigma^{N}\right|} \sum_{i \in \sigma^{N}} \mathbb{E}\left[X_{0}^{i, N}\right]=x_{0} .
$$


Then, for all $n \geq 0$ such that $T_{n}^{N}$ is finite, there exists a possibly infinite real number $\bar{\tau}_{n+1}$ such that the following a.s. limit holds:

$$
\lim _{N \rightarrow \infty} \tau_{n+1}^{N}=\bar{\tau}_{n+1}
$$

In addition, for all $n \geq 0$ and all $t \leq \bar{\tau}_{n+1}$,

- $\mathbb{E}\left[X_{n}^{i, N}(t)\right]=\mathbb{E}\left[X_{n}^{1, N}(t)\right]$, for all $i$ and

- for all sequences of subsets $\sigma^{N}$ of $\{1, \ldots, N\}$, with a cardinal $\left|\sigma^{N}\right|$ that tends to $\infty$ and which is independent of the past throughput process before time $T_{n}^{N}+t$,

$$
\lim _{N \rightarrow \infty} \frac{1}{\left|\sigma^{N}\right|} \sum_{i \in \sigma^{N}} X_{n}^{i, N}(t)=\lim _{N \rightarrow \infty} \frac{1}{\left|\sigma^{N}\right|} \sum_{i \in \sigma^{N}} \mathbb{E}\left[X_{n}^{i, N}(t)\right] \equiv \bar{X}_{n}(t), \quad \text { a.s. }
$$

and

$$
\lim _{N \rightarrow \infty} \frac{1}{\left|\sigma^{N}\right|} \sum_{i \in \sigma^{N}} L_{n}^{i, N}(t)=\lim _{N \rightarrow \infty} \frac{1}{\left|\sigma^{N}\right|} \sum_{i \in \sigma^{N}} \mathbb{E}\left[L_{n}^{i, N}(t)\right] \equiv \bar{L}_{n}(t), \quad \text { a.s. }
$$

with

$$
\begin{aligned}
& \bar{X}_{n}(t)= \begin{cases}\left(\bar{X}_{n}(0)-\frac{\alpha}{R} \frac{2}{\lambda}\right) e^{-\frac{\lambda t}{2}}+\frac{\alpha}{R} \frac{2}{\lambda}, & t<\bar{\tau}_{n+1} \\
\left(1-\frac{p}{2}\right) c, & t=\bar{\tau}_{n+1}\end{cases} \\
& \bar{L}_{n}(t)=\left(\bar{X}_{n}(0)-\frac{\alpha}{R} \frac{2}{\lambda}\right)\left(1-e^{-\frac{\lambda t}{2}}\right)+\frac{\alpha}{R} t, t \leq \bar{\tau}_{n+1}
\end{aligned}
$$

and with $\bar{X}_{0}(0)=x_{0}$ and $\bar{X}_{n}(0)=\left(1-\frac{p}{2}\right)$ for all $n \geq 1$.

The proof is given in Appendix 8.2. Here are a few important remarks on this theorem.

1. The special case $X_{0}^{i, N}=0$ for all $i$ and $n$ is an instance of initial conditions that meet the assumptions of the theorem.

2. Specializing (8) and (9) to the case where $\sigma^{N}$ is the full set, we get that for all $n$ and $t \leq \bar{\tau}_{n+1}$,

$$
\begin{aligned}
\lim _{N \rightarrow \infty} \frac{S_{n}^{N}(t)}{N} & =\lim _{N \rightarrow \infty} \mathbb{E}\left[\frac{S_{n}^{N}(t)}{N}\right]=\bar{X}_{n}(t) \\
\lim _{N \rightarrow \infty} \frac{M_{n}^{N}(t)}{N} & =\lim _{N \rightarrow \infty} \mathbb{E}\left[\frac{M_{n}^{N}(t)}{N}\right]=\bar{L}_{n}(t) .
\end{aligned}
$$

This means that the random variables $\frac{S_{n}^{N}}{N}, \frac{M_{n}^{N}(t)}{N}, \frac{S_{n}^{N}(t)}{N}$ and $\tau_{n+1}^{N}$, become deterministic as $N$ goes to $\infty$, i.e., a large number of sources leads to the deterministic dynamical model described above. This is the essence of the mean field method. 
3. The quantities $\bar{X}_{n}(t), \bar{L}_{n}(t)$ and $\bar{\tau}_{n+1}$ are constant in $n$ for all $n \geq 1$. So, $\bar{X}_{n}(t), \bar{L}_{n}(t)$ and $\bar{\tau}_{n+1}$ are denoted by $\bar{X}(t), \bar{L}(t)$ and $\bar{\tau}$, respectively throughout the rest of this paper.

Now, we are ready to investigate the finiteness of the inter-congestion times. Let

$$
\lambda^{*}=\frac{2}{c} \frac{\alpha}{R} \text {. }
$$

Corollary 1 The inter-congestion times $\bar{\tau}_{1}$ and $\bar{\tau}$ are finite if and only if $\lambda<\lambda^{*}$. In case $\lambda<\lambda^{*}$, we have

$$
\begin{aligned}
\bar{\tau}_{1} & =\frac{2}{\lambda} \log _{e}\left(1+\frac{\lambda\left(1-\frac{x_{0}}{c}\right)}{\lambda^{*}-\lambda}\right) \\
\bar{\tau} & =\frac{2}{\lambda} \log _{e}\left(1+\frac{\lambda p}{2\left(\lambda^{*}-\lambda\right)}\right) .
\end{aligned}
$$

Proof:It is easy to check, for instance using PASTA (Poisson Arrivals See Time Averages, see e.g. [15]) that for all $t, \bar{L}(t) \leq \frac{\lambda c t}{2}$. Thus

$$
\bar{X}(t)=\bar{X}(0)+\frac{\alpha}{R} t-\bar{L}(t) \geq \bar{X}(0)+\left(\frac{\alpha}{R}-\frac{\lambda}{2} c\right) t .
$$

So if $\lambda<\lambda^{*}$, then for all initial conditions less than $c$, the function $\bar{X}(t)$ reaches level $c$ in a finite time since then $\frac{\alpha}{R}-\frac{\lambda}{2} c>0$. The quantity $\bar{\tau}$ in (16) is precisely the solution in $t$ of the equation $\bar{X}(t)=c$ (with a similar result for $\bar{\tau}_{1}$ ).

If $\lambda \geq \lambda^{*}$, then the slope of the function $\bar{X}(t)$ is always less than or equal to 0 as easily checked on (10). Hence $\bar{\tau}_{1}=\infty$.

The physical interpretation of this result is that in the limiting system, it is only if random losses occur rarely enough that congestion play a role in the regulation. The surprising fact is that the conditions under which regulation involves congestion is very simple. We will come back to qualitative implications of this result on the finite population case in $\$ 6$.

\section{Analytical Properties of the Poisson Transmission Error, Rate Independent Synchronization Case}

\subsection{Mean Value of the Throughput of the Asymptotic Model}

From the cycle formula (see [15], Chapter 1), the stationary, continuous time, mean value of the throughput of a session, which we will denote by $\bar{x}$ in what follows, can be calculated by the formula :

$$
\bar{x}=\frac{\int_{0}^{\bar{\tau}} \bar{X}(t) d t}{\bar{\tau}}
$$


From (10), when $\lambda>0$, simple calculations based on the expression for $\bar{X}(t)$ give

$$
\bar{x}=\frac{\alpha}{R} \frac{2}{\lambda}+\frac{1}{\bar{\tau}} \frac{2}{\lambda}\left(\bar{X}(0)-\frac{\alpha}{R} \frac{2}{\lambda}\right)\left(1-e^{-\frac{1}{2} \lambda \bar{\tau}}\right) .
$$

From (6), (14), Corollary 1 and the above equation,

$$
\bar{x}= \begin{cases}c\left(1-\frac{p}{4}\right) & \text { if } \lambda=0 \\ c \frac{\lambda^{*}}{\lambda}-\frac{c p}{2 \log _{e}\left(1+\frac{\lambda p}{2\left(\lambda^{*}-\lambda\right)}\right)} & \text { if } \lambda<\lambda^{*} \\ c \frac{\lambda^{*}}{\lambda} & \text { if } \lambda \geq \lambda^{*} .\end{cases}
$$

Now, we investigate the properties of the function $\bar{x}$.

Theorem $2 \bar{x}$ is an increasing function of $\lambda$ for $\lambda \in\left[0, \lambda^{*}\right]$ and a decreasing function of $\lambda$ for $\lambda \geq \lambda^{*}$, thus $\bar{x}$ achieves its maximal value $c$ at $\lambda=\lambda^{*}$.

The proof is given in Appendix 8.3.

The above theorem means that some transmission losses need to be added in order to get the best average throughput of TCP/droptail. For $\lambda \geq \lambda^{*}$, from (18) we can derive the following relationship: $\bar{x} \geq c(1-\beta p)$ for $\lambda \in\left[\lambda^{*}, \lambda_{\beta}\right]$, where $\beta$ is a constant with $\beta \in\left[0, \frac{1}{p}\right)$ and $\lambda_{\beta}=\lambda^{*} \frac{1}{(1-\beta p)}$. Since $\bar{x} \geq c\left(1-\frac{1}{4} p\right)$ for $\lambda \in\left[0, \lambda^{*}\right]$, this relationship leads to

- $\bar{x} \geq c\left(1-\frac{1}{4} p\right)$ for $\lambda \in\left[0, \lambda_{\frac{1}{4}}\right]$

- $\frac{\lambda_{\frac{1}{4}}}{\lambda_{\beta}}=\frac{4}{3}(1-\beta p)$.

From Theorem 2, we can expect that for other classes of random transmission errors, the mean value of the throughput can be larger than that in the absence of random losses if the throughput of each session can be between $c\left(1-\frac{1}{4} p\right)$ and $c$ during most of the congestion interval. More detailed discussion for this case is given in $\$ 5$.

\subsection{Refinement of the Square Root Throughput Formula}

Let us show how the throughput formula obtained by this approach is actually a refinement to the classical throughput formulas for TCP throughput [10], [11]. To this end, $\alpha$ is replaced by $\frac{1}{R}$.

If there are no congestion epochs, i.e. if $\lambda \geq \lambda^{*}$, we have

$$
\bar{x}=\frac{2}{R^{2} \lambda}=\frac{\sqrt{2}}{R \sqrt{p_{\text {loss }}}},
$$

where $p_{\text {loss }}=\frac{R^{2} \lambda^{2}}{2}$. 
If $\lambda<\lambda^{*}$, the mean number of packets sent by a session over a cycle is $\bar{x} t$. So the packet loss probability $p_{\text {loss }}$ is given by

$$
p_{\text {loss }}=\frac{p+\lambda \bar{\tau}}{\bar{x} \bar{\tau}}=\frac{(\lambda \bar{\tau}+p) R^{2} \lambda^{2}}{2 \lambda \bar{\tau}-c p R^{2} \lambda}
$$

Therefore,

$$
\bar{x}=\frac{2 \sqrt{f(c, p, R, \lambda)}}{R \sqrt{p_{\mathrm{loss}}}}-\frac{c p}{\lambda \bar{\tau}}
$$

where

$$
f(c, p, R, \lambda)=\sqrt{\frac{\lambda \bar{\tau}+p}{2 \lambda \bar{\tau}-c p R^{2} \lambda}} .
$$

We conclude from (19) and (21) that our framework leads to formulas compatible with the classical estimates for TCP throughput ([10], [11]).

\section{The Finite Population Model and Models based on Other Classes of Point Processes}

In this section, we come back to the general framework of $\S 2$. In particular, here, $N$ is finite and the probability that a given flow experiences a loss at a congestion epoch depends on the actual value of the throughput of this flow at this congestion epoch.

We assume that the point processes $\mathcal{L}_{i}, i=1, \ldots, N$, where $\mathcal{L}_{i}$ denotes the point process of the transmission error losses of session $i$, are jointly stationary (see [15]), with finite intensity $\lambda$ and all with the same law (homogeneity assumption). We denote by $E_{L}$ the Palm probability of $\mathcal{L}_{i}$ (this probability does not depend on $i$ by homogeneity).

In what follows, we assume that the dynamics of $\$ 2$ admits a stationary regime and more precisely that the point process $\mathcal{C}$ of congestion epochs is jointly stationary with the point processes $\mathcal{L}_{i}$, $i=1, \ldots, N$. We denote by $E_{C}$ the Palm probability of $\mathcal{C}$ whenever this point process has a positive and finite intensity.

We denote

- by $X(t)$ the stationary throughput of one of the flows at time $t$,

- by $\gamma(y)$ the random variable with value 1 if a flow of throughput $y$ just before some congestion epoch experiences no loss and with value $1 / 2$ otherwise,

- and by $\tau$ the intercongestion time $T_{1}-T_{0}$.

The rate conservation principle (RCP) which applies to all discontinuous stationary processes (see [15], Chapter 1) states that the continuous algebraic growth rate of the process should compensate the algebraic growth of the process at jump times. 


\subsection{Conservation Law for the Case with Congestions}

Assume that the point process $\mathcal{C}$ has a finite intensity $\mu=E_{C}[\tau]^{-1}$. When applying the RCP to the discontinuous stationary process $X(t)$, this gives the following general conservation law:

$$
\lambda \frac{E_{L}[X(0-)]}{2}+\frac{1}{E_{C}[\tau]}\left(c-E_{C}[X(0-) \gamma(X(0-))]\right)=\frac{\alpha}{R}
$$

or equivalently

$$
E_{L}[X(0-)]=\frac{2 \alpha}{\lambda R}-\frac{2\left(c-E_{C}[X(0-) \gamma(X(0-))]\right)}{\lambda E_{C}[\tau]}
$$

\subsection{Conservation Law for the Case without Congestions}

By the same arguments as above, in the case when there are no congestion points at all, then

$$
E_{L}[X(0-)]=\frac{2 \alpha}{\lambda R}
$$

\subsection{Poisson Case}

In the particular case when the loss point processes are Poisson, we know that there are infinitely many congestion epochs when $N$ is finite. In this case, the PASTA property implies that $E_{L}[X(0-)]=$ $\bar{x}$, so that we then have

$$
\bar{x}=\frac{2 \alpha}{\lambda R}-\frac{2\left(c-E_{C}[X(0-) \gamma(X(0-))]\right)}{\lambda E_{C}[\tau]} .
$$

If in addition synchronization is rate independent, then

$$
\bar{x}=\frac{2 \alpha}{\lambda R}-\frac{c p}{\lambda E_{C}[\tau]}
$$

This equation holds for all finite $N$. However, for finite $N$, both $\bar{x}$ and $E_{C}[\tau]$ are unknown. Notice that for $N=\infty$, this is compatible with the second expression in (18).

\subsection{A Sufficient Condition for the Existence of Congestion Epochs}

We now return to the general framework of the beginning of this section.

Lemma 1 If $\lambda<\frac{2 \alpha}{c R}$ and if the underlying point processes are ergodic, there is a.s. an infinite number of congestion epochs.

Proof: The proof is by contradiction. If there are no congestion points at all, then (23) holds. Since largest possible value for the LHS on this equation is $c$, we then have $\lambda \geq \frac{2 \alpha}{c R}$. 


\section{Simulations}

In this section, we illustrate the results of the last sections via direct simulations of the finite population evolution equations of $\$ 2$. We also validate the qualitative behavior of the mean throughput via NS simulation.

In all simulations, we set $c=100$ and $\alpha=\frac{1}{R}=10$, so that the threshold value defined in (14) is $\lambda^{*}=\frac{2}{c} \frac{1}{R^{2}}=2$ for all examples.

\subsection{Poisson Transmission Error Case}

In this example, we use simulation to check Corollary 1 and Theorem 2 and discuss the sensitivity of the throughput when varying the number of sources $N$ and the synchronization rate $p$.

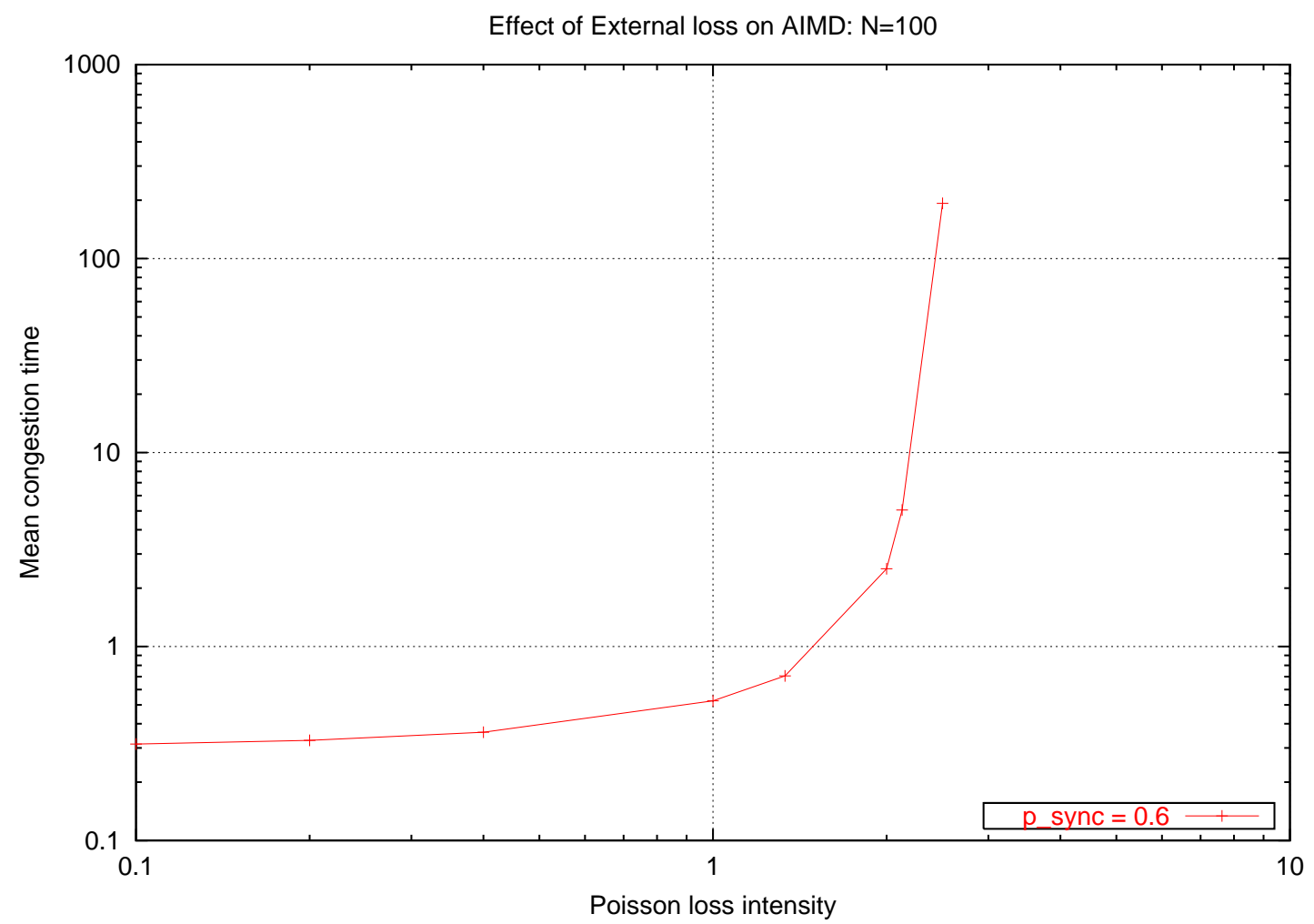

Figure 1: Congestion time vs Transmission loss rate: Poisson case. 


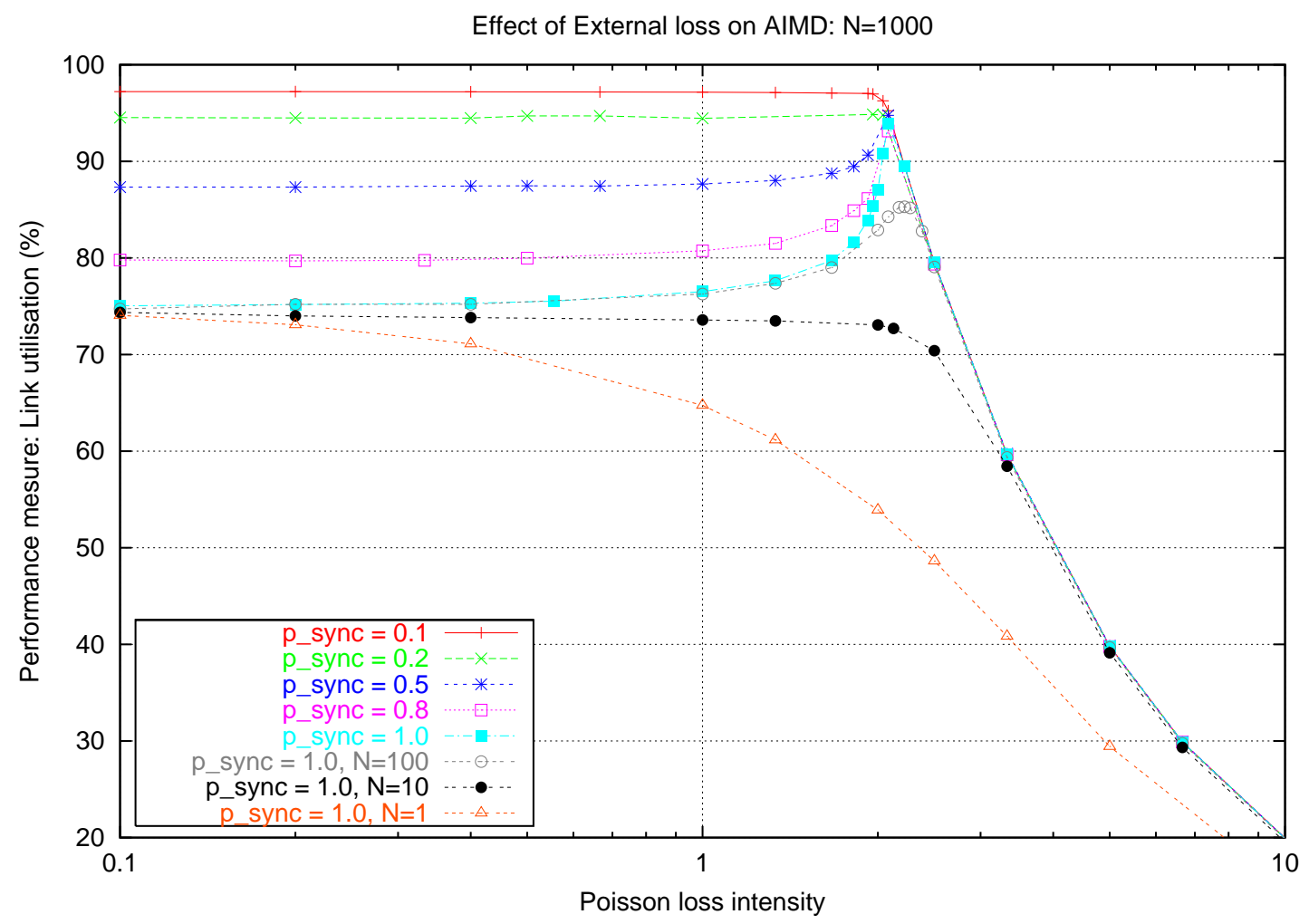

Figure 2: Mean throughput vs Transmission loss rate: Poisson case.

Figure 1 illustrates that the mean inter-congestion time is an increasing function of the transmission loss rate $\lambda$ and drastically increases with $\lambda$ above the threshold, which translates the fact that there are very few congestion epochs above the threshold.

Figure 2 studies the link utilization, which is proportional to the mean throughput in view of homogeneity. This figure shows that the link utilization is an increasing function of $\lambda$ below the threshold $\lambda^{*}=2$ and a decreasing function of $\lambda$ above the threshold. The maximum value of utilization is reached near $\lambda=\lambda^{*}$ indeed. As the number of flows increases, the link utilization curve gets closer and closer to that of the mean throughput as predicted in the asymptotic dynamic model in Theorem 2. This figure also illustrates that the gain in utilization when moving from $\lambda=0$ to $\lambda=\lambda^{*}$ is proportional to the synchronization rate $p$. The gain is $c \frac{p}{4}$ as shown in Theorem 2 . 


\subsection{Other Point Process Transmission Error Case}

In this example, we illustrate what might be the analogues of Corollary 1 and Theorem 2 for other class of point processes as discussed in $§ 5$. For other class of point processes than Poisson, it seems to be difficult to get explicit equations like those in Corollary 1 and Theorem 2. We simulated the case where the error transmission process is a renewal process with Pareto distribution (with parameter equal to 2.5). When comparing this case with the Poisson case, the parameter of the Poisson distribution is chosen in such a way that inter-loss times have the same mean values (equivalently, the transmission error point processes have the same intensity).

Figure 3 shows that the congestion time is again an increasing function of the transmission-error rate below some threshold, so that this fact is not a simple artefact of Poisson point processes. Above this threshold, there are very few congestion epochs.

Figure 4 also seems to indicate that the value of the threshold is different from that of the Poisson case. The threshold of the Pareto case is larger than that of the Poisson case. The fact that when there are no congestions, the average throughput increases when moving from Poisson to Pareto can be explained by the fact that the Pareto law is likely to create more bursty losses. For a fixed mean inter-loss time, a more bursty distribution implies clusters of losses on one side, and very large inter-loss times on the other side. In our model, clusters of losses do not affect too much throughput (several halvings still lead to a positive value) whereas TCP takes advantage of very large inter-loss times thanks to the fact that we did not assume an upper bound on the window size.

\subsection{Throughput vs Transmission Loss Rate: NS Simulation}

As a final example, we consider NS simulation for the effect of the combination of transmission errors and congestion losses on persistent TCP flows sharing a common droptail link. This simulation has the following main differences from the previous ones. First, the synchronization rate cannot be kept constant while varying the transmission loss rate (see Figure 5 for our observation showing the relationship between the total packet loss rate and the transmission packet loss rate in NS simulation). Second, there is a buffer $(B=100)$ in the droptail router which may affect the performance. So, the results in this subsection can in no way be considered a direct analogue of those of the previous two numerical examples. However, one can clearly observe the same qualitative behavior, with an increase of throughput up to the critical value, and then a phase without congestions where throughput decreases with the transmission loss rate as shown in Figure 6. Figure 5 also shows that the total packet loss rate slightly decreases up to the threshold and drastically increases after that. In fact, we can get the maximal throughput when the transmission loss rate is equal to the congestion rate, where the congestion rate is the total loss rate when there is no transmission error.

\section{Conclusions}

In this paper, we proposed a model, called the transmission-error AIMD model, for understanding the dynamical behavior of long lived TCP flows and analyzing their performance in the presence of random packet losses due to both transmission errors and congestion. Based on the transmission- 
Effect of External loss on AIMD: $N=100, p \_s y n c=0.6$

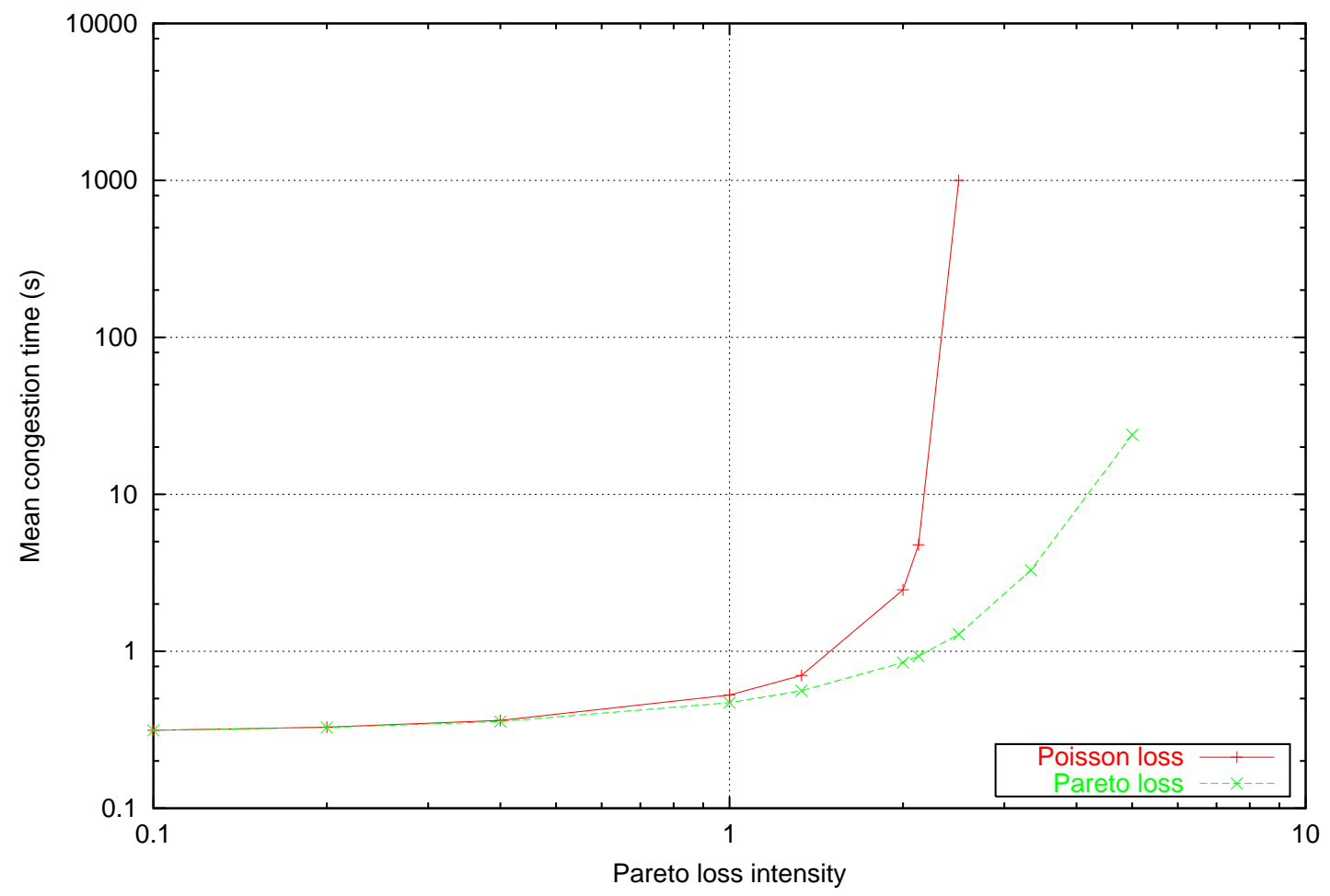

Figure 3: Congestion time vs Transmission loss rate: Pareto case.

error AIMD model, we derived a dynamical system for the large population of TCP sessions in the case of Poisson transmission errors and rate-independent synchronization. From the asymptotic dynamical system, we analyzed the mean throughput of each session and refined the classical throughput formula. We also studied the finite population model and models based on other classes of point processes. Finally, we illustrated our studies via some simulation examples.

This work can be extended to further cases such as heterogeneous TCP flows with different classes of random transmission errors and multiple tail-drop/AQM routers (see our previous papers $[9,12]$ for heterogeneous TCP flows over multiple wired links and [16, 17] for AQM routers.) 


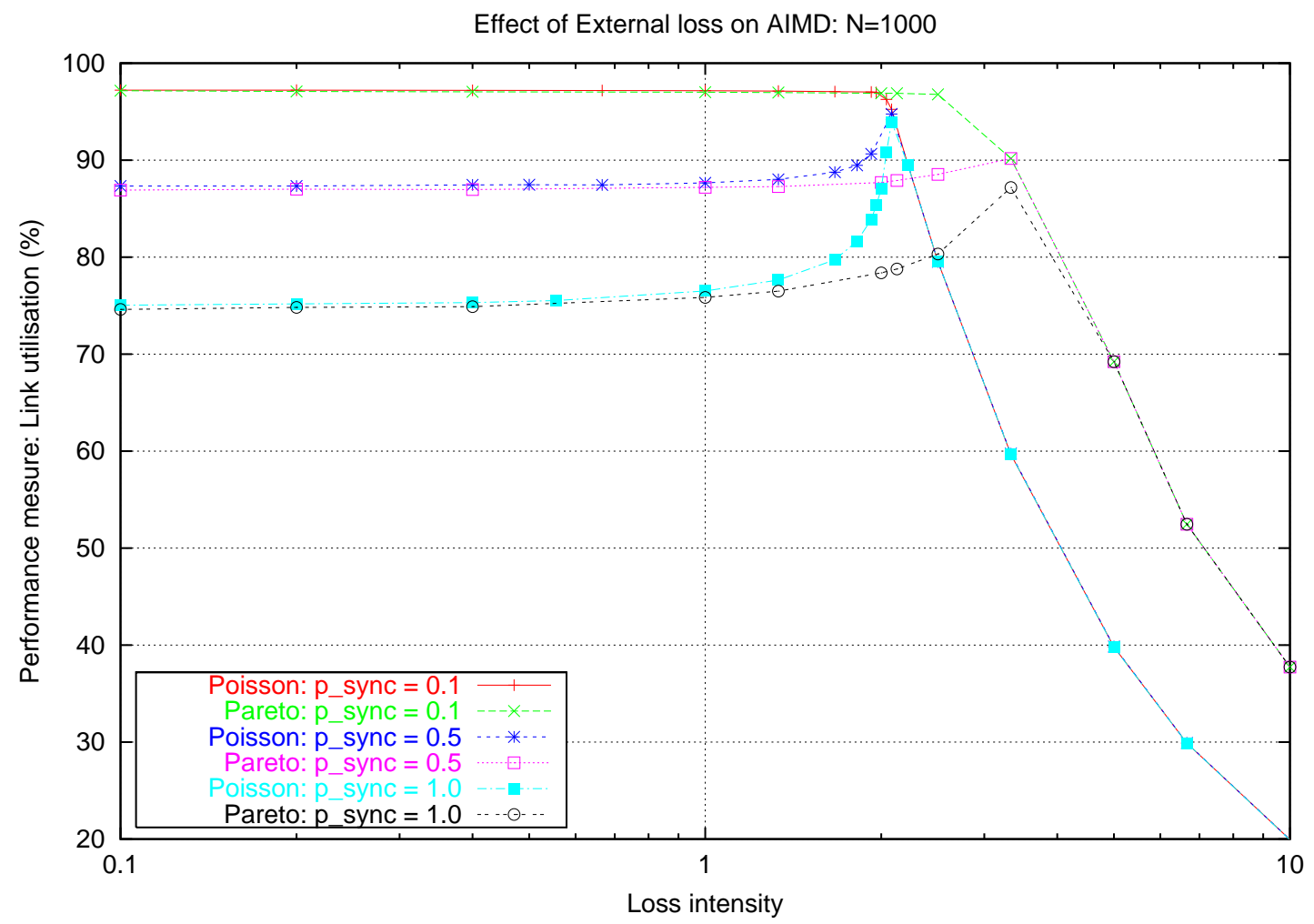

Figure 4: Mean throughput vs Transmission loss rate: Pareto case.

\section{Acknowledgment}

The authors would like to thank Dohy Hong for his contributions to this paper and his help during its preparation.

\section{Appendix}

\subsection{Proof of Equation (5)}

If the $i$-th session experiences $l$ transmission losses in the interval $\left[T_{n}^{N}, T_{n}^{N}+t\right], t<\tau_{n+1}^{N}$, then its throughput is given by

- $\frac{1}{2}\left(X_{n}^{i, N}+\frac{\alpha^{(i)}}{R^{(i)}} \tau_{1}^{i, N}\right)$ at the first random loss; 


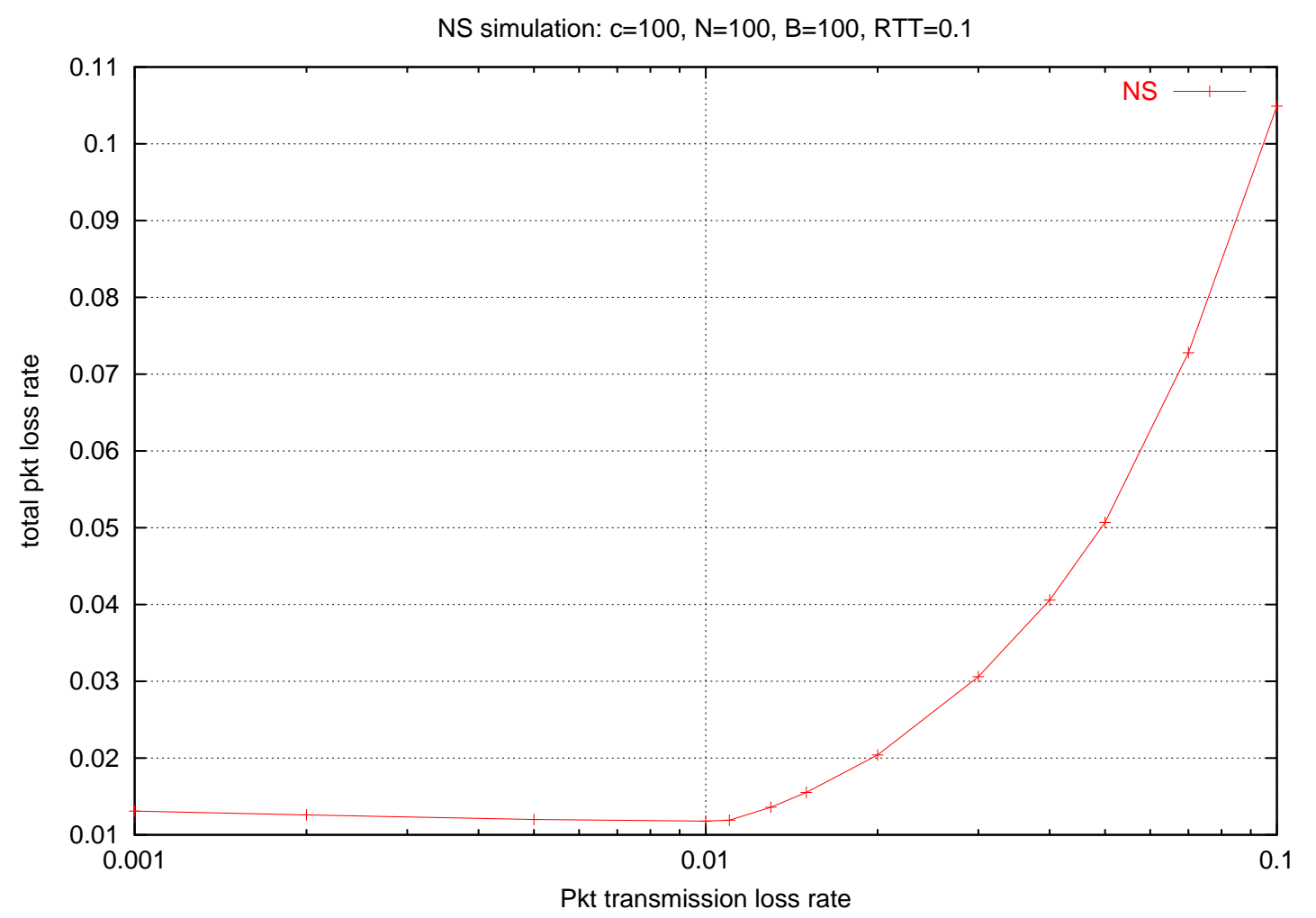

Figure 5: Total loss rate vs Transmission loss rate.

- $\frac{1}{2}\left(\frac{1}{2}\left(X_{n}^{i, N}+\frac{\alpha^{(i)}}{R^{(i)}} \tau_{1}^{i, N}\right)+\frac{\alpha^{(i)}}{R^{(i)}} \tau_{2}^{i, N}\right)$ at the second random loss;

- :

- $\frac{1}{2^{l}} X_{n}^{i, N}+\frac{\alpha^{(i)}}{R^{(i)}} \sum_{k=1}^{l} \frac{1}{2^{l-k+1}} \tau_{k}^{i, N}$ at the $l$-th random loss,

- $\frac{1}{2^{l}} X_{n}^{i, N}+\frac{\alpha^{(i)}}{R^{(i)}} \sum_{k=1}^{l+1} \frac{1}{2^{l-k+1}} \tau_{k}^{i, N}$ at time $t$, 


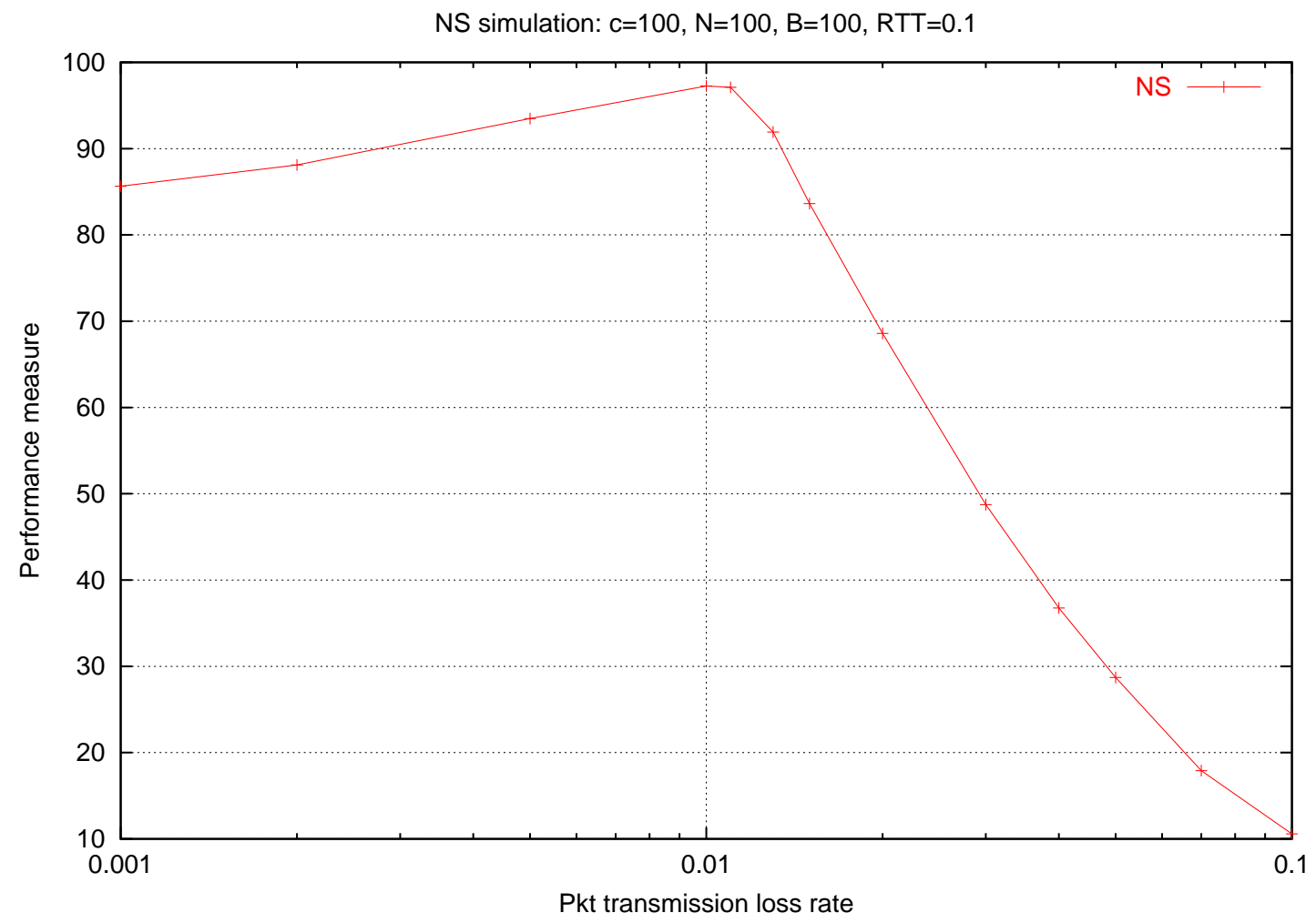

Figure 6: Mean throughput vs Transmission loss rate.

where the variables $\tau_{k}^{i, N} \mathrm{~s}$ are defined as in (5). Thus, if the $n+1$-th congestion epoch takes place later than $T_{n}^{N}+t$, then

$$
\left.X_{n}^{i, N}(t)\right|_{l}=X_{n}^{i, N} \frac{1}{2^{l}}+\frac{\alpha^{(i)}}{R^{(i)}} t-\frac{\alpha^{(i)}}{R^{(i)}} \sum_{k=1}^{l+1} \tau_{k}^{i, N}\left(1-\frac{1}{2^{l-k+1}}\right),
$$

so that

$$
\left.L_{n}^{i, N}(t)\right|_{l}=X_{n}^{i, N}\left(1-\frac{1}{2^{l}}\right)+\frac{\alpha^{(i)}}{R^{(i)}} \sum_{k=1}^{l+1} \tau_{k}^{i, N}\left(1-\frac{1}{2^{l-k+1}}\right),
$$

which completes the proof of (5). 


\subsection{Proof of Theorem 1}

The proof is by induction. We take $T_{0}^{N}=0$. The induction assumption is that

- $\mathbb{E}\left[X_{n}^{i, N}\right]=\mathbb{E}\left[X_{n}^{1, N}\right]$ for all $i$ and

- for all sequences of subsets $\sigma^{N}$ of $\{1, \ldots, N\}$, such that $\left|\sigma^{N}\right|$ tends to $\infty$ and such that this set is independent of the past throughput process before time $T_{n}^{N}$,

$$
\lim _{N \rightarrow \infty} \frac{1}{\left|\sigma^{N}\right|} \sum_{i \in \sigma^{N}} X_{n}^{i, N}=\lim _{N \rightarrow \infty} \frac{1}{\left|\sigma^{N}\right|} \sum_{i \in \sigma^{N}} \mathbb{E}\left[X_{n}^{i, N}\right]= \begin{cases}x_{0} \text { a.s. } & \text { if } n=0 \\ \left(1-\frac{1}{2} p\right) c \text { a.s. } & \text { if } n \geq 1 .\end{cases}
$$

This assumption holds for $n=0$ by the assumption of Theorem 1 . We first prove via a sequence of lemmas that if this induction assumption holds true for $n$, then it holds true for $n+1$. For the sake of clear exposition, we will actually only prove that the desired property holds for $n+1$ for the whole set $\{1, \ldots, N\}$. The extension to any subset with the appropriate properties is obtained by a mere rephrasing of the proofs below.

In a first step, we consider the system with transmission errors only.

Let $s_{l}^{N}(t)$ denote the set of sessions that experience $l$ transmission losses on the time interval $\left[T_{n}^{N}, T_{n}^{N}+t\right], t>0$. The empirical mean of the throughput drop on this interval, namely $\frac{M_{n}^{N}(t)}{N}$, can be represented as

$$
\begin{aligned}
\frac{M_{n}^{N}(t)}{N} & =\frac{1}{N}\left(\left.\sum_{l \geq 0} \sum_{i \in s_{l}^{N}(t)} L_{n}^{i, N}(t)\right|_{l}\right) \\
& =\sum_{l \geq 0} \frac{\left|s_{l}^{N}(t)\right|}{N} \frac{\left.\sum_{i \in s_{l}^{N}(t)} L_{n}^{i, N}(t)\right|_{l}}{\left|s_{l}^{N}(t)\right|},
\end{aligned}
$$

where $0 / 0$ is 0 by convention.

For all $N \geq 1$, the collection of real numbers

$$
\mu_{l}^{N}=\frac{\left|s_{l}^{N}(t)\right|}{N}, \quad l \in \mathbb{N},
$$

is a probability measure on $\mathbb{N}$ (the non-negative integers). From the fundamental theorem of statistics, the probability measures $\mu^{N}$ converges weakly to the Poisson probability measure $q$ of parameter $\lambda t$ on $\mathbb{N}$.

For all $N \geq 1$, let $g^{N}: \mathbb{N} \rightarrow \mathbb{R}$ be the function:

$$
g_{l}^{N}=\frac{\left.\sum_{i \in s_{l}^{N}(t)} L_{n}^{i, N}(t)\right|_{l}}{\left|s_{l}^{N}(t)\right|} .
$$

We prove in Lemma 3 below that under the induction assumption, for all fixed $l \geq 1$, there exists a bounded function $g: \mathbb{N} \rightarrow \mathbb{R}^{+}$such that for all $l, \lim _{N \rightarrow \infty} g_{l}^{N}=g_{l}$ a.s. Let $A$ denote the upper bound on $g$. 
Lemma 2 The following interchange of limit and sum is licit:

$$
\lim _{N \rightarrow \infty} \sum_{l} \mu_{l}^{N} g_{l}^{N}=\sum_{l} \lim _{N \rightarrow \infty} \mu_{l}^{N} g_{l}^{N}=\sum_{l} q_{l} g_{l} \quad \text { a.s. }
$$

Proof: For all $J$ and $N$,

$$
\left|\sum_{l} \mu_{l}^{N} g_{l}^{N}-\sum_{l} q_{l} g_{l}\right| \leq h(N, J)
$$

with

$$
h(N, J)=\left|\sum_{l<J} \mu_{l}^{N} g_{l}^{N}-\sum_{l<J} q_{l} g_{l}\right|+A \sum_{l \geq J} q_{l}+\sum_{l \geq J} \mu_{l}^{N} g_{l}^{N} .
$$

Pick any $\epsilon>0$; there exists $J$ such that $\sum_{l \geq J} q_{l} \leq \epsilon$.

Since for all fixed $l, \mu_{l}^{N} g_{l}^{N}$ tends to $q_{l} g_{l}$, then there exists a random integer $K_{1}$ such that for all $N \geq K_{1}$

$$
\left|\sum_{l<J} \mu_{l}^{N} g_{l}^{N}-\sum_{l<J} q_{l} g_{l}\right| \leq \epsilon .
$$

In addition

$$
\begin{aligned}
\sum_{l \geq J} \mu_{l}^{N} g_{l}^{N} & =\frac{\sum_{l \geq J}\left|s_{l}^{N}(t)\right|}{N} \frac{\sum_{l \geq J} \sum_{i \in s_{l}^{N}(t)} L_{n}^{i, N}}{\sum_{l \geq J}\left|s_{l}^{N}(t)\right|} \\
& =\left(\sum_{l \geq J} \mu_{l}^{N}\right) \frac{\sum_{l \geq J} \sum_{i \in s_{l}^{N}(t)} L_{n}^{i, N}}{\sum_{l \geq J}\left|s_{l}^{N}(t)\right|}
\end{aligned}
$$

Notice that since $X_{n}^{i, N}(t) \geq 0$ for all $t$, it follows from (2) that

$$
\left.\sum_{i \in s_{l}^{N}(t)} L_{n}^{i, N}(t)\right|_{l} \leq \sum_{i \in s_{l}^{N}(t)}\left(X_{n}^{i, N}+\frac{\alpha}{R} t\right) .
$$

Let

$$
G_{n}^{N}=\frac{\sum_{l \geq J} \sum_{i \in s_{l}^{N}(t)}\left(X_{n}^{i, N}+\frac{\alpha}{R} t\right)}{\sum_{l \geq J}\left|s_{l}^{N}(t)\right|} .
$$

From the induction assumption and from the fact that $\lim _{N \rightarrow \infty}\left|\cup_{l \geq J} s_{l}^{N}(t)\right| \rightarrow \infty$,

$$
\lim _{N \rightarrow \infty} G_{n}^{N}= \begin{cases}x_{0}+\frac{\alpha}{R} t & \text { if } n=0 \\ \left(1-\frac{1}{2} p\right) c+\frac{\alpha}{R} t & \text { if } n \geq 1\end{cases}
$$

$\operatorname{RR} n^{\circ} 4953$ 
Hence there exists a $K_{2}$ such that for all $N \geq K_{2}, \lim _{N \rightarrow \infty} G_{n}^{N} \leq \frac{\alpha}{R} t+\max \left(x_{0},\left(1-\frac{1}{2} p\right) c\right)$.

Furthermore, since $\mu^{N}$ converges weakly to $q$, there exists a random integer $K_{3}$ such that for all $N \geq K_{3}$,

$$
\left|\sum_{l \geq J} q_{l}-\sum_{l \geq J} \mu_{l}^{N}\right| \leq \epsilon, \text { so that } \sum_{l \geq J} \mu_{l}^{N} \leq 2 \epsilon .
$$

So for all $N \geq \max \left(K_{1}, K_{2}, K_{3}\right), h(N, J) \leq \epsilon\left[1+A+2 \frac{\alpha}{R} t+2 \max \left(x_{0},\left(1-\frac{1}{2} p\right) c\right)\right]$. Since $\epsilon$ is arbitrary, (26) is proved.

Lemma 3 Under the foregoing assumptions, if $n=0$

$$
\lim _{N \rightarrow \infty} g_{l}^{N}=x_{0}\left(1-\frac{1}{2^{l}}\right)+\frac{\alpha}{R} \sum_{k=1}^{l+1} \frac{t}{l+1}\left(1-\frac{1}{2^{l-k+1}}\right), \quad \text { a.s. }
$$

whereas for $n \geq 1$

$$
\lim _{N \rightarrow \infty} g_{l}^{N}=\left(1-\frac{1}{2} p\right) c\left(1-\frac{1}{2^{l}}\right)+\frac{\alpha}{R} \sum_{k=1}^{l+1} \frac{t}{l+1}\left(1-\frac{1}{2^{l-k+1}}\right), \quad \text { a.s. }
$$

Proof: Since for all $l$, the set $s_{l}^{N}(t)$ is independent of the past throughput process before $T_{n}^{N}$ and in particular of the random vector $X_{n}^{i, N}$, from the induction assumption, we have

$$
\lim _{N \rightarrow \infty} \frac{\sum_{i \in s_{l}^{N}(t)} X_{n}^{i, N}}{\left|s_{l}^{N}(t)\right|}= \begin{cases}x_{0} & \text { if } n=0 \\ \left(1-\frac{1}{2} p\right) c & \text { if } n \geq 1\end{cases}
$$

Let us now show that in addition,

$$
\lim _{N \rightarrow \infty} \frac{\sum_{i \in s_{l}^{N}(t)} \tau_{k}^{i, N}}{\left|s_{l}^{N}(t)\right|}=\frac{t}{l+1}
$$

for all $t$ in a positive range, and all $k \in[1, l+1]$.

Since the point processes of transmission losses after $T_{n}^{N}$ are independent Poisson point processes with intensity $\lambda$, the strong law of large number implies that

$$
\lim _{N \rightarrow \infty} \frac{\sum_{i \in s_{l}^{N}(t)} \tau_{k}^{i, N}}{\left|s_{l}(t)\right|}=\mathbb{E}\left[\tau_{k}^{i, N} \mid i \in s_{l}\right]
$$

for any $k \in[1, l+1]$. We now show that

$$
\mathbb{E}\left[\tau_{1}^{i, N} \mid i \in s_{l}\right]=\mathbb{E}\left[\tau_{2}^{i, N} \mid i \in s_{l}\right]=\cdots=\mathbb{E}\left[\tau_{l+1}^{i, N} \mid i \in s_{l}\right]=\frac{t}{l+1} .
$$


It is well known that given the number of points $l$ of a Poisson point process in the interval $[0, t]$, these $l$ points are independent and uniformly distributed between 0 and $t$. From this fact, we can easily get that $\mathbb{E}\left[\tau_{1}^{i, N} \mid i \in s_{l}\right]=\frac{t}{l+1}$. Let us now show that in fact, $\mathbb{E}\left[\tau_{k}^{i, N} \mid i \in s_{l}\right]$ for $k \in[2, l+1]$.

Let $v$ be a real number between 0 and $t$, and $U_{j}=\sum_{k=1}^{j} \tau_{k}^{i, N}$. Then, given $t$ and $l$, the distribution function of the random variable $U_{j}$ is given by

$$
P\left(U_{j} \leq v\right)=\sum_{k=j}^{l} \frac{l !}{(l-k) ! k !}\left(\frac{v}{t}\right)^{k}\left(\frac{t-v}{t}\right)^{l-k} .
$$

Thus, the density function for $U_{j}$ is given by

$$
f(u)=\frac{l !}{(l-j) ! j !} \frac{j}{t}\left(\frac{u}{t}\right)^{j-1}\left(\frac{t-u}{t}\right)^{l-j} .
$$

From the density function, we can get $\mathbb{E}\left[\tau_{j+1}^{i, N} \mid i \in s_{l}\right]=\mathbb{E}\left[U_{j+1}-U_{j}\right]$ for all $j \in[1, l-1]$ as follows.

$$
\begin{aligned}
\mathbb{E}\left[U_{j+1}-U_{j}\right] & =\int_{0}^{t} \mathbb{E}\left[U_{j+1}-U_{j} \mid U_{j}=u\right] f\left(U_{j}=u\right) d u \\
& =\int_{0}^{t} \frac{t-u}{l-j+1} \frac{l !}{(l-j) ! j !} \frac{j}{t}\left(\frac{u}{t}\right)^{j-1}\left(\frac{t-u}{t}\right)^{l-j} d u \\
& =\int_{0}^{t} \frac{t}{l+1} \frac{(l+1) !}{(l+1-j) ! j !} \frac{j}{t}\left(\frac{u}{t}\right)^{j-1}\left(\frac{t-u}{t}\right)^{l+1-j} d u \\
& =\frac{t}{l+1} \text { for all } j \in[1, l-1] .
\end{aligned}
$$

Since $\mathbb{E}\left[\tau_{k}^{i, N} \mid i \in s_{l}\right]=\frac{t}{l+1}$ for all $k \in[1, l], \mathbb{E}\left[\tau_{l+1}^{i, N} \mid i \in s_{l}\right]=\frac{t}{l+1}$. (5))

The proof of the lemma is now immediately concluded from the following fact (deduced from

$$
g_{l}^{N}=\frac{\left.\sum_{i \in s_{l}^{N}(t)} X_{n}^{i, N}\right|_{l}}{\left|s_{l}^{N}(t)\right|}\left(1-\frac{1}{2^{l}}\right)+\frac{\alpha}{R} \sum_{k=1}^{l+1} \frac{\sum_{i \in s_{l}^{N}(t)} \tau_{k}^{i, N}}{\left|s_{l}^{N}(t)\right|}\left(1-\frac{1}{2^{l-k+1}}\right)
$$

and from $(27,28)$.

A direct consequence of Lemma 2 is that if $n \geq 1$,

$$
\lim _{N \rightarrow \infty} \frac{M_{n}^{N}(t)}{N}=\sum_{l} q_{l}\left[\left(1-\frac{p}{2}\right) c\left(1-\frac{1}{2^{l}}\right)+\frac{\alpha}{R} \sum_{k=1}^{l+1} \frac{t}{l+1}\left(1-\frac{1}{2^{l-k+1}}\right)\right], \text { a.s. }
$$

with a similar conclusion for $n=0$ when replacing $\left(1-\frac{1}{2} p\right) c$ by $x_{0}$. By the same arguments, if $n \geq 1$,

$$
\lim _{N \rightarrow \infty} \frac{S_{n}^{N}(t)}{N}=\sum_{l} q_{l}\left[\left(1-\frac{p}{2}\right) c\left(\frac{1}{2^{l}}\right)+\frac{\alpha}{R} t-\frac{\alpha}{R} \sum_{k=1}^{l+1} \frac{t}{l+1}\left(1-\frac{1}{2^{l-k+1}}\right)\right], \quad \text { a.s. }
$$


with a similar conclusion for $n=0$ when replacing $\left(1-\frac{1}{2} p\right) c$ by $x_{0}$.

When denoting by $\bar{X}_{n}(t)$ the deterministic limit of $\frac{S_{n}^{N}(t)}{N}$ and by $\bar{L}_{n}(t)$ the deterministic limit of $\frac{M_{n}^{N}(t)}{N}$, we can summarize the last results as (10) and (11), respectively.

Let us now come back to the true system where congestion may take place. Since $\tau_{n+1}^{N}=$ $\inf \left\{t\right.$ s.t $\left.S_{n}^{N}(t)=c N\right\}$, and since the system without congestions and the true system coincide until $\tau_{n+1}^{N}$, the fact that the function $\frac{S_{n}^{N}(t)}{N}$ considered above tends to the deterministic and continuous function $\bar{X}(t)$, as $N$ tends to infinity, implies that $\tau_{n+1}^{N}$ tends to a deterministic limit that will be denoted by $\bar{\tau}_{n+1}$. In the rest of this proof, we assume that $\bar{\tau}_{n+1}$ is finite.

By simple continuity arguments, it is easy to check that for all $t<\bar{\tau}_{n+1}$, the limiting behaviors of the system without congestions and of the true systems coincide, which proves (8) and (9) for $t<\bar{\tau}_{n+1}$ and for $\sigma^{N}=\{1, \ldots, N\}$.

As mentioned above, it is easy to extend this result to the case with subsets $\sigma^{N}$ such that $\left|\sigma^{N}\right|$ tends to $\infty$ and such that this set is independent of the past throughput process before time $T_{n}^{N}+t$.

Lemma 4 Under the foregoing assumptions, when denoting by $f(t-)$ the left hand limit of the function $f$ at point $t$, the following a.s. limit holds:

$$
\lim _{N \rightarrow \infty} \frac{1}{N} S_{n}^{N}\left(\tau_{n+1}^{N}-\right)=\lim _{N \rightarrow \infty} \frac{1}{N} \mathbb{E}\left[S_{n}^{N}\left(\tau_{n+1}^{N}-\right)\right]=\bar{X}\left(\bar{\tau}_{n+1}-\right) .
$$

Proof:The proof is based on continuity arguments. Pick $\epsilon>0$. Taking $t$ in an appropriate left neighborhood of $\bar{\tau}_{n+1}$, we have

$$
\left|\bar{X}(t)-\bar{X}\left(\bar{\tau}_{n+1}-\right)\right|<\epsilon
$$

by continuity. In addition, this neighborhood can be chosen such that

$$
\lim _{N \rightarrow \infty}\left|\frac{1}{N} S_{n}^{N}\left(\tau_{n+1}^{N}-\right)-\frac{1}{N} S_{n}^{N}(t)\right|<\epsilon .
$$

The last property is obtained by considering the subset $\sigma^{N}$ of flows that experience no losses in the interval $\left[t, \tau_{n+1}^{N}\right)$. This set has a cardinality that tends to infinity and it is independent of the past throughput process before $T_{n}^{N}+t$. When $\tau_{n+1}^{N}-t<\eta$,

$$
\left|\frac{1}{N} S_{n}^{N}\left(\tau_{n+1}^{N}-\right)-\frac{1}{N} S_{n}^{N}(t)\right| \leq \eta \frac{\alpha}{R}+\frac{N-\left|\sigma^{N}\right|}{N} \frac{1}{N-\left|\sigma^{N}\right|} \sum_{i \notin \sigma^{N}} X_{n}^{(i, N)}(t) .
$$

When $N$ is large enough, $\frac{N-\left|\sigma^{N}\right|}{N}$ is close to the probability that a Poisson point process has one or more points in an interval of length $\eta$ which can be made arbitrarily small. In addition, from what was proved above, when $N$ is large enough, $\frac{1}{N-\left|\sigma^{N}\right|} \sum_{i \notin \sigma^{N}} X_{n}^{(i, N)}(t)$ tends to a finite constant. Hence (31) is proved. Combining (30), (31) and the fact that

$$
\left|\frac{1}{N} S_{n}^{N}(t)-\bar{X}(t)\right|<\epsilon
$$


for $N$ large enough allows us to conclude the proof for that the first limit of the lemma is equal to $\bar{X}\left(\bar{\tau}_{n+1}\right)$. The proof of the convergence of the expectations is similar.

Lemma 5 Under the foregoing assumptions, the following a.s. limit holds:

$$
\lim _{N \rightarrow \infty} \frac{1}{N} S_{n}^{N}\left(\tau_{n+1}^{N}\right)=\lim _{N \rightarrow \infty} \frac{1}{N} \mathbb{E}\left[S_{n}^{N}\left(\tau_{n+1}^{N}\right)\right]=\bar{S}\left(\bar{\tau}_{n+1}\right) .
$$

Proof: The proof is immediate from the last lemma when introducing the subset $\sigma^{N}$ of flows that experience a loss at time $T_{n+1}^{N}$. We use here the fact that the synchronization is rate independent

The extension of the last lemma to the case of more general subsets of the set $\{1, \ldots, N\}$ completes the proof of the induction.

\subsection{Proof of Theorem 2}

First, we show that the average throughput $\bar{x}$ is a continuous function of $\lambda \geq 0$. It is easy to see that

$$
\lim _{\lambda \rightarrow \lambda^{*}} \frac{1}{\log _{e}\left(1+\frac{\lambda p}{2\left(\lambda^{*}-\lambda\right)}\right)}=0
$$

so $\bar{x}$ is continuous at $\lambda=\lambda^{*}$. Next, we show that

$$
\lim _{\lambda \rightarrow 0}\left(c \frac{\lambda^{*}}{\lambda}-\frac{c p}{2 \log _{e}\left(1+\frac{\lambda p}{2\left(\lambda^{*}-\lambda\right)}\right)}\right)=c\left(1-\frac{p}{4}\right) .
$$

Let $a=\frac{\lambda p}{2\left(\lambda^{*}-\lambda\right)}$. Then,

$$
c \frac{\lambda^{*}}{\lambda}-\frac{c p}{2 \log _{e}\left(1+\frac{\lambda p}{2\left(\lambda^{*}-\lambda\right)}\right)}=c+\frac{c p}{2}\left(\frac{1}{a}-\frac{1}{\log _{e}(1+a)}\right) .
$$

Let $b=\log _{e}(1+a)$. Then, $1+a=e^{b}=1+b+\frac{b^{2}}{2 !}+\frac{b^{3}}{3 !}+\cdots$ and thus

$$
\frac{1}{b}-\frac{1}{a}=\frac{1}{a}\left(\frac{b}{2 !}+\frac{b^{2}}{3 !}+\cdots\right)=\frac{1}{\left(b+\frac{b^{2}}{2 !}+\frac{b^{3}}{3 !}+\cdots\right)}\left(\frac{b}{2 !}+\frac{b^{2}}{3 !}+\cdots\right) .
$$

Since $b \rightarrow 0$ as $\lambda \rightarrow 0$, we have $\lim _{\lambda \rightarrow 0}\left(\frac{1}{b}-\frac{1}{a}\right)=\frac{1}{2}$. This completes the proof of (35).

Now, we prove that $\bar{x}$ in (18) is a strictly increasing function of $\lambda$ for $\lambda \in\left(0, \lambda^{*}\right)$. We have

$$
\frac{\partial \bar{x}}{\partial \lambda}=\frac{2}{R^{2} \lambda^{2}}\left(\frac{1}{\log _{e}(1+a)}\right)^{2} \frac{1}{(1+a)}\left[a^{2}-(1+a)\left(\log _{e}(1+a)\right)^{2}\right]>0
$$

for all $a>0$ (i.e., $\lambda \in\left(0, \lambda^{*}\right)$ ). The last relationship can be proved as follows. Let

$$
f(a)=a^{2}-(1+a)\left(\log _{e}(1+a)\right)^{2} .
$$


Then, we have

$$
\frac{d f(a)}{d a}=2 a-\left(\log _{e}(1+a)\right)^{2}-2 \log _{e}(1+a)
$$

and

$$
\frac{d^{2} f(a)}{(d a)^{2}}=2 a-\left(\log _{e}(1+a)\right)^{2}-2 \log _{e}(1+a)=\frac{2}{(1+a)}\left(a-\log _{e}(1+a)\right) .
$$

Since $\frac{d^{2} f(a)}{(d a)^{2}}>0$ for all $a>0$ and $\frac{d f(a)}{d a}=0$ when $a=0, \frac{d f(a)}{a}>0$ for all $a>0$. Similarly, since $\frac{d f(a)}{d a}>0$ for all $a>0$ and $f(a)=0$ when $a=0, f(a)>0$ for all $a>0$.

\section{References}

[1] C. Barakat, E. Altman, and W. Dabbous, "On TCP performance in a heterogeneous network: a survey," IEEE Communications Magazine, vol. 38, 2000.

[2] V. Tsaoussidis and I. Matta, "Poen issues on TCP for mobile computing," Journal of Wireless Communications and Mobile Computing-Special Issue on Reliable Transport Protocols for Mobile Computing, vol. 2, no. 1, 2002.

[3] H. Balakrishnan, V. N. Padmanabhan, S. Seshan, and R. H. Katz, "A comparison of mechanisms for improving TCP performance over wireless links," IEEE/ACM Transactions on Networking, vol. 5, pp. 756-769, December 1997.

[4] A. Biaz and N. Vaidya, "Discriminating congestion losses from wireless losses using interarrival times at the receiver," in IEEE Symposium (ASSET'99), 1999.

[5] J. Liu, I. Matta, and M. Crovella, "End-to-end interface of loss nature in a hybrid wired/wireless environment," in Proceedings of the workshop (WiOpt'03), March 2003.

[6] A. Bakre and B. R. Badrinath, "I-TCP: Indirect TCP for mobile hosts," in Proc. 15th Int. Conf. Distributed Computing Syst. (ICDCS), May 1995.

[7] T. Henderson and R. H. Katz, "Transport protocols for internet-compatible satellite networks," IEEE Journal on Selected Areas in Communications, vol. 17, pp. 326-344, 1999.

[8] F. Baccelli and D. Hong, "AIMD, fairness and fractal scaling of TCP traffic," in Proceedings of IEEE Infocom, April 2002.

[9] F. Baccelli and D. Hong, "Interaction of TCP flows as billiards," in Proceedings of IEEE Infocom, April 2003.

[10] M. Mathis, J. Semke, J. Mahdavi, and T. Ott, "The macroscopic behavior of the TCP congestion avoidance algorithm," ACM Computer Communication Review, vol. 27, July 1997.

[11] J. Padhye, V. Firoiu, D. Towsley, and J. Kurose, "Modeling TCP throughput: a simple model and its empirical validation," in Proceedings of ACM/SIGCOMM, 1998. 
[12] F. Baccelli and D. Hong, "Flow level simulation of large IP networks," in Proceedings of IEEE Infocom, April 2003.

[13] P. Brémaud, Markov Chains: Gibbs Fields, Monte Carlo Simulation and Queues. Springer Verlag, 1999.

[14] D. Hong and D. Lebedev, "Many TCP user asymptotic analysis of the aimd model," tech. rep., INRIA, Paris, France, 2001, RR 4229.

[15] F. Baccelli and P. Brémaud, Elements of Queueing Theory. Springer Verlag, 2002.

[16] K. B. Kim and S. H. Low, "Design of receding horizon AQM in stabilizing TCP with multiple links and heterogeneous delays," in Proc. of 4th Asian Control Conference, vol. WA-1, (Singapore), 2002.

[17] K. B. Kim and S. H. Low, "Analysis and design of AQM based on state-space models for stabilizing TCP," in Proc. of American Control Conference, vol. WA08-4, (Denver, Cololado), 2003. 
Unité de recherche INRIA Rocquencourt Domaine de Voluceau - Rocquencourt - BP 105 - 78153 Le Chesnay Cedex (France)

Unité de recherche INRIA Lorraine : LORIA, Technopôle de Nancy-Brabois - Campus scientifi que 615, rue du Jardin Botanique - BP 101 - 54602 Villers-lès-Nancy Cedex (France)

Unité de recherche INRIA Rennes : IRISA, Campus universitaire de Beaulieu - 35042 Rennes Cedex (France)

Unité de recherche INRIA Rhône-Alpes : 655, avenue de l'Europe - 38330 Montbonnot-St-Martin (France)

Unité de recherche INRIA Sophia Antipolis : 2004, route des Lucioles - BP 93 - 06902 Sophia Antipolis Cedex (France) 